\title{
Beobachtungen zum Beutespektrum von Zitterspinnen (Pholcidae)
}

\author{
Helge UHLENHAUT
}

Abstract: Observations on the prey of pholcids (Pholcidae)

Key words: Pholcus ssp., prey, Bavaria, Germany

Im folgenden möchte ich eine kleine Studie zum Beutespektrum unserer heimischen Pholcus-Arten und zur Arachnofauna in einem Wohnhaus im Frankenwald (Bayern) zum besten geben.

Während eines Aufenthalts bei meinen Schwiegereltern fielen mir bei der Benutzung der Toilette die auf dem Fußboden liegenden eingesponnenen Beutepakete der unter der Decke hängenden Zitterspinnen auf. Bei einer ersten Sichtung der in einem Schnappdeckelgläschen gesammelten Beutereste zeigte sich schon, dass ein großer Teil aus eingesponnenen Spinnen bestand. Nun ist ja bekannt, dass die einheimischen PholcusArten regelmäßig Spinnen, insbesondere aber die großen in Gebäuden lebenden Tegenaria-Arten erbeuten, die von mir eingesammelten Beutereste stammten jedoch zumindest teilweise offensichtlich von Individuen anderer Arten.

\section{METHODE UND UNTERSUCHUNGSRAUM}

Da unsere einheimischen Pholcus-Arten ihre Beute mit ihren sehr kleinen Cheliceren bekanntlich nicht durchkneten, sondern lediglich aussaugen, konnten die nahezu unbeschädigten Chitinhüllen der erbeuteten Spinnen, soweit sie adult waren, bis zur Art bestimmt werden. Dieser Umstand und die Tatsache, dass außer den Pholcus-Netzen keinerlei andere Gespinste auffindbarwaren, stellte auch einigermaßen sicher, dass die aufgesammelten Beutereste ausschließlich den Zitterspinnen zuzuordnen waren. Da mich schon die Ergebnisse der ersten Auswertung in Erstaunen setzten, wurden 
in der Folge über einen Zeitraum von insgesamt 16 Monaten (September 1998 bis Juni 1999, Mai bis Oktober 2000) sämtliche in oben genannter Toilette auffindbaren Beutepakete gesammelt und unter dem Binokular ausgewertet. Da nicht sicher abgeschätzt werden konnte, wie weit die Akzeptanz für mein arachnologisches Interesse an der häuslichen PholcusPopulation ging, wurde die Sammeltätigkeit, meine Schwiegermutter möge es verzeihen, nicht im Familienkreis diskutiert. Allerdings ließ sich deshalb auch nachträglich leider nicht feststellen, wieviele Beutereste während des Untersuchungszeitraums Putzaktivitäten zum Opfer gefallen sind.

Tab. 1: Erbeutete Spinnenarten

Tab. 1: List of spiders captured by Pholcus ssp.

\begin{tabular}{|c|c|c|c|}
\hline Artname & $\mathbf{N}$ & Artname & $\mathbf{N}$ \\
\hline Alopecosa cuneata & $10^{\circ}, 3$ 우 & Linyphia hortensis & $10^{\pi}$ \\
\hline Alopecosa taeniata & $40^{x} 0^{x}$ & Linyphia triangularis & 19 \\
\hline Amaurobius fenestralis & $10^{*}$ & Metellina mengei & $30^{\pi}$ \\
\hline Araneus diadematus & $10^{\pi}$ & Metellina segmentata & $10^{*}, 39 \%$ \\
\hline Aulonia albimana & $20^{x} \sigma^{x}$ & Nesticus cellulanus & $10^{\pi}$ \\
\hline Callobius claustrarius & $20^{*} 0^{*}$ & Pardosa lugubris & $10^{*}, 1+9$ \\
\hline Cicurina cicur & $10^{\pi}$ & Pholcus opilionides & $10^{\pi}$ \\
\hline Clubiona coerulescens & $10^{\pi}$ & Pholcus phalangioides & $20^{x} 0^{x}, 2$ 우우 \\
\hline Clubiona terrestris & $10^{x}, 1+9$ & Pisaura mirabilis & $20^{7} \sigma^{*}$ \\
\hline Coelotes terrestris & $70^{\circ} 0^{\circ}, 399$ & Porrhomma microphthalmum & $1+$ \\
\hline Drassodes lapidosus & $10^{*}$ & Tegenaria atrica & 1 우 \\
\hline Drassodes pubescens & $10^{*}$ & Tegenaria silvestris & $50^{x} 0^{x}, 6$ 우우 \\
\hline Dysdera erythrina & $10^{\pi}, 2$ 우 & Trochosa terricola & $10^{\pi}, 2$ 우아 \\
\hline Enoplognatha ovata & 19 & Troxochrus nasutus & $10^{\pi}, 4$ 우우 \\
\hline Harpactea lepida & $1+$ & Walckenaeria dysderoides & $10^{x}$ \\
\hline Harpactea rubicunda & $10^{*}$ & Walckenaeria mitrata & $20^{\pi}$ \\
\hline Histopona torpida & $100^{x} 0^{x}, 3$ 우 우 & Zora spinimana & 19 \\
\hline Labulla thoracica & $20^{\pi} 0^{\pi}$ & juvenile Lycosidae & 8 \\
\hline Lepthyphantes alacris & 19 & juvenile Linyphiidae & 2 \\
\hline Lepthyphantes leprosus & $10^{\pi}, 1$ 우 & juvenile Agelenidae & 3 \\
\hline Lepthyphantes pallidus & $20^{x} 0^{x}$ & juvenile Pholcidae & 5 \\
\hline
\end{tabular}


Tab. 2: Erbeutete Insekta, Crustacea, Myriapoda und andere Arachnida Tab. 2: Insecta, Crustacea, Myriapoda and Arachnida (other than Araneae) captured by Pholcus ssp.

$\begin{array}{lr}\text { Insecta Insekten } & \text { N } \\ \text { Coleoptera Käfer } & 1 \\ \text { Carabidae, Abax parallelepipedus } & 1 \\ \text { Carabidae, Poecilus versicolor } & 2 \\ \text { Carabidae, Pterostichus niger } & 1 \\ \text { Carabidae, Pterostichus oblongopunctatus } & 3 \\ \text { Elateridae } & 2 \\ \text { Staphylinidae, Ocypus sp. } & 1 \\ \text { Silphidae, Phosphuga atrata } & 1 \\ \text { Chrysomelidae } & 1 \\ \text { Curculionidae, cf Phyllobius argentatus } & 3 \\ \text { Curculionidae, cf Otiorhynchus sulcatus } & \\ \text { Hymenoptera Hautflügler } & 1 \\ \text { Formicidae, Camponotus ligniperda } & 1 \\ \text { Formicidae, Tapinoma erraticum } & 1 \\ \text { Formicidae, Myrmica rubra } & \\ \text { Blattaria Schaben } & 1 \\ \text { Ectobius lapponicus } & \\ \text { Lepidoptera Schmetterlinge } & 1 \\ \text { Geometridae } & \\ \text { Dermaptera Ohrwürmer } & \\ \text { Forficula auricularia } & \\ \text { Diptera Zweiflügler } & \\ \text { Brachycera } & \\ \text { Nematocera, Tipulidae } & \\ \text { Raphidioptera Kamelhalsfliegen } & \\ \text { Raphidia sp. } & \\ \text { Planipennia Netzflügler } & \\ \text { Chrysopa vulgaris } & \\ & \end{array}$

N Crustacea Krebstiere N Isopoda Asseln

1 Porcellio montanus 23

1 Porcellio scaber 8

2 Oniscus asellus 3

Chilopoda Hundertfüßer

Lithobius cf. forficatus

Diplopoda Tausendfüßer

Glomeris conspersa

Polydesmus sp.

Julidae

Arachnida Spinnentiere

Opiliones Weberknechte

Paranemastoma quadripunctatum 1

Mitopus morio

Das Wohnhaus meiner Schwiegereltern liegt einzeln an einem südwestexponierten Hang des Grümpeltals, ca $12 \mathrm{~km}$ nördlich von Kronach und ca 550 m üNN (TK 5634). Der unmittelbare Umgriff des Anwesens besteht teilweise aus offenen Flächen aufanstehendem Schiefergestein und teilweise aus Mischwald. Die oben genannte Toilette (ca. $4 \mathrm{~m}^{2}$ ) ist Teil einer relativ gering frequentierten Einliegerwohnung und liegt im Erdgeschoß des Gebäudes. Die von den Zitterspinnen erbeuteten Tiere sind wohl zum größeren Teil durch das meist gekippte Fenster in das Gebäude eingedrungen, der andere Teil gehört zu den permanenten Mitbewohnern. 


\section{ERGEBNISSE}

Wie die beiden Tabellen der ausgewerteten Beutereste zeigen, wurden neben den Spinnen auch andere Arthropoden von den Zitterspinnen gefangen. Die Webspinnen machten jedoch sowohl bezüglich der Artenwie auch der Individuenzahlen den größten Anteil aus. So konnten unter den Beuteresten mindestens 38 Spinnenarten (118 Individuen) nachgewiesen werden. Nun richtet sich bei den meisten Spinnen die Beutezusammensetzung nach dem Angebot, und in anderen Gebäuden oder auch schon in anderen Wohnräumen würden ganz andere Ergebnisse erzielt werden. Bemerkenswert erscheinen mir dennoch in diesem Zusammenhang sowohl die Anzahl der oben genannte Toilette frequentierenden Spinnenarten, als auch die Tatsache, daß die meisten der unter den Beuteresten gefundenen Arten wie z.B. Troxochrus nasutus oder Alopecosa taeniata nicht gerade als synanthrop gelten. Die beiden letztgenannten Arten sind, nebenbei bemerkt, in der Bayerischen Roten Liste unter der Gefährdungskategorie $4 \mathrm{~S}$ aufgeführt (BLICK \& SCHEIDLER 1992).

Während meiner Aufsammlungen konnte ich immer etwa 6 adulte Pholcus-Individuen im „Untersuchungsraum" zählen. Zwischenzeitlich wurden immer wieder auch juvenile Individuen unterschiedlicher Entwicklungsstadien und in schwankender Dichte beobachtet. Daß es sich hier um beide bei uns heimischen Zitterspinnenarten Pholcus phalangioides und $P$. opilionides handelte, wurde erst klar, als in den Beutepaketen adulte Vertreter beider Arten gefunden wurden. Offensichtlich sind Zitterspinnen keineswegs voreinander sicher. Mit 13 Individuen wurde Histopona torpida am häufigsten von den Zitterspinnen erbeutet, insgesamt liegt ein gewisser Schwerpunkt bei den Ageleniden (38 Ind.) und Lycosiden (23 Ind.).

Wie die Tabelle 2 verdeutlicht, sind die außer den Webspinnen erbeuteten Arthropoden den unterschiedlichsten Gruppen zuzuordnen. So reicht das Beutespektrum von stark gepanzerten Käfern wie z.B. Phosphuga atrata bis zu filigranen Fluginsekten wie den Tipuliden, es umfaßt aber auch Arten mit wirksamen Wehrsekreten wie die Laufkäfer oder Tausendfüßer. Auffällig war, dass die von den Zitterspinnen erbeuteten Käfer sehr viel dicker in Spinnseide eingewickelt waren als andere Beutetiere wie z.B. Spinnen oder Asseln, was den Schluss zuläßt, daß Käfer sich effektiver oder anhaltender wehren. Unter den nicht zu den Webspinnen zu rechnenden Beuteresten nahmen die Asseln mit 34 von 102 Individuen ein Drittel ein. 


\section{SCHLUSSFOLGERUNG}

Insgesamt wurden in einem etwa $4 \mathrm{~m}^{2}$ großen Raum in 16 Monaten 220 Beuterestpakete aufgesammelt. Das bedeutet, die nicht abschätzbaren Verluste durch Reinigungsarbeiten einmal außer Acht gelassen, dass fast jeden zweiten Tag ein größeres Beutetier von den Zitterspinnen überwältigt werden konnte. Da während meiner durchaus auch nächtlichen Besuche im „Untersuchungsraum" nie potentielle Beutetiere beobachtetwerden konnten, muss man wohl davon ausgehen, dass die Zitterspinnen in der Lage sind ihren eng umgrenzten Lebensraum äußerst effektiv zu nutzen, sodass kaum ein Gliederfüßler die Chance erhält, diese Toilette im Haus meiner Schwiegereltern, sollte er denn erst einmal dorthin gelangt sein, wieder lebend zu verlassen.

\section{LITERATUR}

BLICK, T. \& M.SCHEIDLER (1992): Rote Liste gefährdeter Spinnen (Araneae) Bayerns. In: Bayerisches Landesamt für Umweltschutz (Hrsg.): Rote Liste gefährdeter Tiere Bayerns.-Beiträge zum Artenschutz 15. Schr.R. Bayer. LA Umweltschutz 111: 56-66; München.

Helge UHLENHAUT, Stadtparkring 18a, D-08523 Plauen e-mail: Helge.Uhlenhaut@t-online.de 